\title{
Monitoring of partial and full venous outflow obstruction in a porcine flap model using laser speckle contrast imaging
}

Johan Zotterman, Max Bergkvist, Fredrik Iredahl, Erik Tesselaar and Simon Farnebo

\section{Linköping University Post Print}

\section{Tweet}

N.B.: When citing this work, cite the original article.

Original Publication:

Johan Zotterman, Max Bergkvist, Fredrik Iredahl, Erik Tesselaar and Simon Farnebo, Monitoring of partial and full venous outflow obstruction in a porcine flap model using laser speckle contrast imaging, 2016, Journal of Plastic, Reconstructive \&amp; Aesthetic Surgery, (69), 7, 936-943.

http://dx.doi.org/10.1016/j.bjps.2016.02.015

Copyright: Elsevier

http://www.elsevier.com/

Postprint available at: Linköping University Electronic Press

http://urn.kb.se/resolve?urn=urn:nbn:se:liu:diva-130059

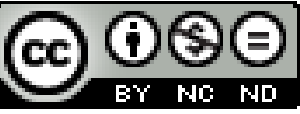




\section{Monitoring of Partial and Full Venous Outflow}

\section{Obstruction in a Porcine Flap Model using Laser Speckle}

\section{Contrast Imaging}

Johan Zötterman $^{1}$, Max Bergkvist ${ }^{1}$, Fredrik Iredahl ${ }^{2}$, Erik Tesselaar ${ }^{3}$, and Simon Farnebo ${ }^{1}$

${ }^{1}$. Department of Hand and Plastic Surgery and Burns, Department of Clinical and Experimental Medicine, Linköping University, Linköping, Sweden.

2. Department of Clinical and Experimental Medicine, Linköping University, Linköping, Sweden.

3. Department of Radiation Physics, Department of Clinical and Experimental Medicine, Linköping University, Linköping, Sweden.

Corresponding author:

Johan Zötterman

Department of Clinical and Experimental Medicine

Linköping University

58185 Linköping, Sweden

tel. +46703370524

E-mail. johan.zotterman@regionostergotland.se 


\section{Abstract}

\section{Background:}

In microsurgery, there is a demand for more reliable methods of postoperative monitoring of free flaps, especially with regard to tissue threatening obstructions of the feeding arteries and draining veins. In this study, we evaluated laser speckle contrast imaging and laser Doppler flowmetry to assess their possibilities to detect partial and full venous outflow obstruction, as well as full arterial occlusion, in a porcine flap model.

\section{Methods:}

Cranial gluteal artery perforator flaps (CGAP) were raised and arterial and venous blood flow to and from the flaps was monitored using ultrasonic flow probes. The venous flow was altered with an inflatable cuff to simulate partial and full (50\% and 100\%) venous obstruction and arterial flow was completely obstructed using clamps. The flap microcirculation was monitored using Laser Speckle Contrast Imaging (LSCI) and Laser Doppler Flowmetry (LDF).

\section{Results:}

Both LDF and the LSCI detected significant changes in flap perfusion. After partial (50\%) venous occlusion, perfusion decreased from baseline, LSCI: $63.5 \pm 12.9$ PU ( $p=0.01)$, LDF $31.3 \pm 15.7$ ( $\mathrm{p}=0.64)$. After $100 \%$ venous occlusion, a further decrease in perfusion was observed: LSCI $54.6 \pm 14.2$ PU ( $<$ < 0.001) and LDF 16.7 \pm 12.8 PU ( $<0.001)$. After release of the venous cuff, LSCI detected a return of the perfusion to a level slightly, but not significantly, below baseline level $70.1 \pm 11.5$ PU ( $\mathrm{p}=0.39)$ while the LDF signal returned to 
a level not significant from baseline $36.1 \pm 17.9$ PU ( $p>0.99)$. Perfusion during $100 \%$ arterial occlusion decreased significantly as measured with both methods, LSCI: $48.3 \pm 7.7$ (PU, p < 0.001) and LDF: $8.5 \pm 4.0$ PU (p < 0.001). During 50\% and 100\% venous occlusion, LSCI showed a $20 \%$ and $26 \%$ intersubject variability (CV \%), respectively, compared to $50 \%$ and $77 \%$ for LDF.

\section{Conclusions:}

LSCI offers sensitive and reproducible measurements of flap microcirculation and seems more reliable in detecting decreases in blood perfusion caused by venous obstruction. Also, it allows for perfusion measurements in a relatively large area of flap tissue. This may be useful in identifying areas of the flap with compromised microcirculation during and after surgery.

\section{Keywords:}

free flaps; venous occlusion; arterial occlusion; laser Doppler; laser speckle contrast imaging 


\section{Introduction}

Microsurgical free-tissue transfer is a well-established technique to cover defects after trauma and cancer surgery. The major cause of flap failure is venous thrombosis, which leads to a decrease of the blood drainage from the flap and a stasis and subsequent congestion of blood in the tissue. This mostly occurs within the first two days postoperatively and during this time span it's vital for the surgeon and other health staff to regularly check up on the viability of the flap $[1,2]$.

The success rate for most types of microsurgical free tissue transfers is very high. Still, follow ups show that some patients will need reoperation due to compromised flap viability and that a few percent of the flaps are lost due to flap failure [1, 3, 4].

One challenge has therefore been to find a reliable way to monitor the viability of the flap with a high degree of sensitivity and specificity. Surgeons still much rely on clinical controls like colour changes of the skin, changes in temperature and capillary blink. However, a number of different methods has evolved over the years to allow for more reliable and user independent surveillance of flap viability [5].

One of the most commonly used techniques for monitoring blood flow in microvascular flaps is Laser Doppler Flowmetry (LDF), which measures the microvascular perfusion by combining the concentration and velocity of red blood cells in the tissue. This method is limited by its small measurement volume and the measured perfusion value is therefore subject to the large inherent heterogeneity of the microcirculation of the flap tissue, which in turn can produce false high or low perfusion values [6-11]. 
Laser Speckle Contrast Imaging (LSCI) is a camera-based technique that illuminates an area of tissue with divergent $785 \mathrm{~nm}$ laser light and analyses the interference pattern of the light that is scattered from the tissue. This makes it possible to measure perfusion in an area of tissue. The method has been used both in experimental settings to measure blood perfusion after controlled tissue provocations, including post-occlusive reactive hyperaemia, and clinically to assess perfusion in scald burns [12-15].

Most studies on flap perfusion have been focusing on arterial obstruction, rather than venous obstruction. This is even though venous congestion has been shown to occur more frequently and to cause more deleterious effects on the tissue than arterial ischemia [16]. As vessel thrombosis is likely to progress from partial to full occlusion, we designed this study to address partial as well as full venous obstruction. We used a porcine flap model that allows for controlled venous occlusion with the aim to compare LSCI measurements to single point measurements with LDF $[17,18]$. The CGAP flap was designed to consistently include an area of compromised perfusion, in order to assess if LSCI could detect spatial variations in perfusion depending on the distance from the pedicle.

\section{Materials and Methods}

\section{Animals}

Five mixed breed pigs (mean age 4 months, 45 kg, Swedish Landrace pigs) were used for the study. They were pre-anesthetized with Dexdomitor $0.1 \mathrm{mg} / \mathrm{kg}$, Zoletil $5 \mathrm{mg} / \mathrm{kg}$ and atropine $0.05 \mathrm{mg} / \mathrm{kg}$. Anaesthesia was withheld with Pentobarbital sodium $8 \mathrm{mg} / \mathrm{kg} / \mathrm{h}$ and fentanyl $0.5 \mu \mathrm{g} / \mathrm{kg} / \mathrm{h}$ dissolved in Ringer's acetate given continuously intravenously with motorized infuser along with crystalloid fluids (Ringer acetate). Body temperature, blood 
pressure, heart rate and oxygen saturation were monitored during the whole procedure. After the interventions and measurements had been performed on one side, the animal was turned and the procedure was repeated on the contralateral side. The pigs were finally euthanized with a mix of Pentobarbital sodium and 70\% ethanol without regaining consciousness.

\section{Surgery}

A 12 x15 cm fascio-cutaneous island flap based on the cranial gluteal artery perforant (CGAP) were raised from the buttocks of each pig (Figure 1). The flap was dissected along the surface of the muscle, including skin, subcutaneous tissue and muscle fascia in the flap. The pedicle of the flap, containing the perforator artery along with comitant veins was carefully isolated and the sensory nerve cut. The comitant veins were separated from the artery and one of them was ligated. Then, perivascular flow probes (Transonic Precision Perivascular Flow Probe MA-2PSB Transonic Systems Inc. 34 Dutch Mill Road, Ithaca, NY 14850 USA) were placed around the vessels, after which the corners of the flap were sutured to the corners of the defect and wet swabs were placed on uncovered wound surfaces to keep them moist.

An inflatable vascular occluder (Norfolk Medical Products Inc., 7350 N. Ridgeway, Skokie, Illinois 60076 USA) was placed around the vein, distal to the flow probe, and fixated to nearby tissue with sutures. A $2 \mathrm{ml}$ syringe was used to inflate the cuff with saline to control the amount of occlusion applied to the vein.

\section{Equipment}

Transonic perivascular flow probes (Transonic Precision Perivascular Flow Probe MA2PSB Transonic Systems Inc. 34 Dutch Mill Road, Ithaca, NY 14850 USA) were used to monitor the flow in the supplying vessels. The first probe was placed at the base of the artery 
and the second at the base of the vein. Both were fixated with sutures to the surrounding tissue. The probes were connected to a Transonic TS420 Perivascular Flow Meter Module.

A Laser Speckle Contrast Imager (PeriCam PSI System, Perimed AB, Järfälla, Sweden) was placed approximately $20-25 \mathrm{~cm}$ above the surface of the flap to measure the perfusion in different areas of the skin of the flap. In short, LSCI measures perfusion illuminating the tissue with a divergent laser beam with a wavelength of $785 \mathrm{~nm}$. This creates a speckle pattern over the illuminated area. A CMOS camera captures the speckle image while another captures a conventional colour image of the measured area. The principle of the measurement technique of LSCI has been previously described in detail $[12,13]$. The image size was set to correspond to a $10 \mathrm{x} 15 \mathrm{~cm}$ area of skin and the distance between the camera and the skin was kept between 20 and $25 \mathrm{~cm}$. The acquisition rate was set to 21 images/s. With each measurement, the perfusion data from 42 consecutive images were averaged, resulting in a total measurement time of $2 \mathrm{~s}$ for each image. The spatial resolution of the perfusion image is $0.2 \mathrm{~mm} / \mathrm{pixel}$ at a measurement distance of $20-25 \mathrm{~cm}$.

In addition to LSCI, perfusion was also monitored continuously using two laser Doppler flow probes (Probe 45781-1, Perimed AB, Järfälla, Sweden) connected to a Laser Doppler Monitor (PeriFlux, Perimed AB, Järfälla, Sweden). The probes have a fibre separation of 0.25 $\mathrm{mm}$ and measure perfusion at a depth of about $0.5 \mathrm{~mm}$.

Skin temperature was monitored continuously using a thermistor taped to the skin surface. All measurement devices were calibrated according to the instructions of the manufacturers before the experiments. 


\section{Measurements}

After the surgery was performed, the flap was raised and the baseline measurements started. Baseline hemodynamic and ventilatory readings, flap arterial flow, flap venous outflow, and skin temperature were measured every 5 minutes. Skin perfusion was continuously measured using LDF (two different probes, one probe heated to 40 degrees Celsius and one unheated) and LSCI. LSCI images were acquired every 5 minutes during baseline and every minute during occlusion.

After 30 minutes of baseline measurements, the flap outflow vein was partially occluded to obtain a flap arterial flow around $50 \%$ of the mean baseline flap arterial flow. After 30 minutes of partial occlusion, the flap vein was clamped with a removable microvascular clamp to achieve $100 \%$ occlusion. The clamp was removed after 30 minutes and a 40 minutes venous recovery phase was initiated. The protocol ended with 30 minutes arterial occlusion with the vascular clamp applied to the flap artery followed by 30 minutes of arterial recovery.

\section{Statistics}

LSCI images were processed using the system's analysis software (PSIWin, Perimed, Järfälla, Sweden). In each image, three circular regions of interest were selected within the flap, one proximal to the pedicle, one centrally and one distal (Figure 1a). They were positioned approximately $2 \mathrm{~cm}$ apart and had a diameter of approximately $3 \mathrm{~cm}$. For each image, the average perfusion in each ROI was calculated. Laser Doppler perfusion data was collected with an interval of 5 minutes. 
Shapiro-Wilk normality tests were performed to confirm that the data was no inconsistent with a Gaussian distribution. All data is in text and tables are presented as mean \pm SD. Two-way analyses of variance for repeated measures with Sidak's multiple comparisons test were performed to test whether changes from baseline were significant, for the respective measurement techniques. Intersubject variability was assessed by percent coefficient variability. Statistical calculations were done using GraphPad Prism version 6.0 for Mac OS X (GraphPad Software, San Diego California USA, “www.graphpad.com”). For all analyses, probabilities of less than 0.05 were accepted as significant.

\section{Results}

Blood pressure and heart frequency were kept stable throughout the protocol in all animals. No changes in temperature were seen in any of the raised flaps.

Mean arterial and venous blood flow through the pedicle, as well change in mean skin perfusion measured by LDF and LSCI are presented in Figure 2. During baseline, mean arterial and venous blood flow through the pedicle as measured using the flow probes was $13.34 \pm 0.75$ $\mathrm{ml} / \mathrm{min}$ and $13.37 \pm 0.72 \mathrm{ml} / \mathrm{min}$, respectively. Perfusion in the skin during baseline was 76.8 \pm 9.9 PU (LSCI) and $36.6 \pm 17.3 \mathrm{PU}$ (LDF). After partial (50\%) venous occlusion, mean arterial blood flow was reduced to $9.39 \pm 0.67 \mathrm{ml} / \mathrm{min}$ while mean venous blood flow was $5.24 \pm 0.36$ $\mathrm{ml} / \mathrm{min}$. Perfusion as measured with LSCI was reduced to $63.5 \pm 12.9 \mathrm{PU}(\mathrm{p}=0.01)$, while no change was seen using LDF $31.3 \pm 15.7$ ( $p=0.64$ ). After $100 \%$ venous occlusion, a further decrease mean arterial blood flow (4.99 $\pm 0.66 \mathrm{ml} / \mathrm{min})$, mean venous blood flow $(0.03 \pm 0.02$ $\mathrm{ml} / \mathrm{min}$ ) was observed. The perfusion was also reduced compared to baseline, both as measured using LSCI 54.6 \pm 14.2 PU ( $<$ 0.001) and LDF 16.7 \pm 12.8 PU $(p<0.001)$. After release of 
the venous cuff, blood flow through the pedicle returned to basal levels (arterial flow $14.31 \pm$ 0.62, venous flow $13.00 \pm 0.49 \mathrm{ml} / \mathrm{min}$ ) while perfusion, as measured using LSCI, returned to a level slightly, but not significantly, below baseline level, $70.1 \pm 11.5 \mathrm{PU}(\mathrm{p}=0.39)$ while perfusion, as measured using LDF, returned to a level not significantly different from baseline, $36.1 \pm$ 17.9 PU (p > 0.99) (Table 2). After total arterial occlusion, arterial flow was effectively stopped $(0 \mathrm{ml} / \mathrm{min})$ while venous flow was $0.15 \pm 0.03 \mathrm{ml} / \mathrm{min}$. Perfusion during $100 \%$ arterial occlusion decreased significantly as measured with both methods, LSCI: $48.3 \pm 7.7$ (PU, p < 0.001) and LDF: $8.5 \pm 4.0 \mathrm{PU}(\mathrm{p}<0.001)$. During all provocations, perfusion as measured with LSCI had a lower intersubject variability (\% CV) compared to perfusion as measured with LDF (LSCI, baseline.

LSCI images from one representative flap showing typical perfusion images during the six different phases of the protocol are shown in Figure 3. The LSCI images show clear differences in false-colour scale between the different phases of the experiment, associated with changes in perfusion. During all phases, a downward perfusion gradient from the pedicle towards the distal part of the flap can easily be recognized as a gradual change in colour from the left to the right side of the LSCI images. This is further exemplified in the time course of the perfusion in three different ROIs in this particular flap, one in the well-perfused part of the flap (ROI 1), one in the middle (ROI 2) and one in the less-perfused, distal part of the flap (ROI 3). During all phases, the perfusion in ROI 3 is lower than in ROI 1 and ROI 2.

In Figure 4, the mean skin perfusion from the well vascularized part of the flap (ROI 1) were compared to perfusion in the poorly vascularized part of the graft (ROI 3). During all phases of the experiment, the perfusion was lower in the poorly vascularised part of the flap than in the well vascularised part. In the poorly vascularised part of the flap, a significant change in perfusion compared with baseline was only seen during complete arterial occlusion $(\mathrm{p}=0.01)$, 
while no significant changes in perfusion were seen during partial $(p=0.10)$ or complete $(p=0.12)$ venous occlusion. In the well vascularised part of the flap, significant changes in perfusion compared with baseline were seen during both partial $(\mathrm{p}=0.007)$ and complete $(\mathrm{p}=0.01)$ venous occlusion as well as during arterial occlusion $(\mathrm{p}=0.001)$.

\section{Discussion}

Early identification of vascular occlusion in flaps is important as it increases flap salvage. LDF is still the most commonly used method for postoperative monitoring of free flaps, but it is limited by the fact that it only measures perfusion in a single point in the flap. The aim of the study has been to investigate LSCI as an alternative to LDF for the detection of partial and total venous occlusion. We modified a previously established porcine model where alterations in arterial and venous blood flow can reproducibly be altered and closely monitored. An advantage of the model is the similarity of skin features between human and pig as well as the ability to raise a large flap based on a single vessel suited for adequate measurements [19]. Another advantage of the model is that the flap size allows for a zone with compromised circulation, in the distal part of the flap. This enables us to assess how LSCI functions both in well vascularized as well as poorly vascularized tissue.

The main finding of this study is that LSCI can detect regional changes in skin perfusion in the flap after partial and complete venous obstruction. We observed significant decreases in perfusion both during $50 \%$ and complete venous occlusion with LSCI, while changes in perfusion seen with LDF during venous occlusion were less consistent. This result is consistent with results from our previous studies, in which LSCI has been shown to have a higher reproducibility with measuring changes in perfusion after exsanguination, arterial and venous occlusion of the forearm, and during post occlusive reactive hyperaemia (PORH) (unpublished 
results). This difference in sensitivity between LSCI and LDF is most likely explained by the higher variability seen with LDF (measured as \% coefficient of variation), which has also been found in previous studies and is related to the heterogeneity in the perfusion in the skin [11], which considerably affects the LDF results. Placement of the probe will thus greatly affect all LDF measurements whereas LSCI offers the possibility to assess skin perfusion over larger areas. We chose to place the LDF probe consistently in the centre of the well vascularised part of the flap, which is in accordance with how the probe is used in our clinic for postoperative monitoring of flaps. Recordings from this placement will however not give representative data from less vascularized parts of the flap.

We observed an even stronger reduction in skin perfusion during complete arterial occlusion than during both partial and complete venous occlusion. However the perfusion did not reduce to zero, and local variations in perfusion were still seen in the flap during complete arterial occlusion. One possible explanation for this is that the remaining perfusion is caused by a venous backflow during arterial occlusion. Also, the perfusion signal in LSCI is related to both the concentration and the velocity of red blood cells, the remaining perfusion seen during arterial occlusion may also be caused by preserved differences in the concentration of red blood cells during stasis.

In our study we were unable to see any significant postoperative reactive hyperaemia after arterial occlusion. An earlier study, in which Laser Doppler Perfusion Imaging (LDPI) was used to monitor radial forearm flaps peri-operatively showed a significant hyperaemia after reperfusion [9]. The difference between the results in our study and the previous study may be caused by differences in occlusion time [20], or by the fact that we applied a venous occlusion before the arterial occlusion, which could have affected the vascular reactivity. Finally, differences in measurement techniques could be responsible for the difference seen between studies. 
The fact that LSCI provides a spatial perfusion map over the entire flap makes it possible to identify the poorly vascularised zones, something that LDF cannot do. For example, there was a difference in LSCI baseline values between areas with good (ROI 1) and compromised circulation (ROI 3). Also, we observed a tendency for LSCI to give a less significant change in areas that have a compromised circulation at baseline, although the trends are similar. Therefore, is seems that for post-operative monitoring, the mean perfusion in the entire flap, or in the central zone of the flap should be monitored.

When we compared the maximal hyperaemic response after total arterial occlusion between the well vascularised and poorly vascularised areas, we could in the LSCI data further see that perfusion increased from 48.1 to $86.1 \mathrm{AU}$ in ROI 1, which corresponds to a 79 percent relative increase whereas perfusion in ROI 3 increased from 29.4 to $47.1 \mathrm{AU}$, which only corresponds to a $60 \%$ relative increase. That is, the maximum response after arterial occlusion is substantially lower in areas where the LSCI baseline measurements indicate a lower perfusion in the tissue. This also corresponds to the findings of the previously mentioned study [9], in which the typical post occlusive hyperaemia was absent in one flap that was discarded based on clinical observations.

It is thus possible that LSCI can be used peri-operatively, or in prolonged study protocols, during certain types of flap surgery where only some parts of the raised flap is used for the reconstruction, and the rest of the flap is discarded. This decision has traditionally been based on the surgeon's experience, and clinical findings during surgery. The LSCI measurements might offer support for the surgeon in this decision. Finally, it would be interesting to follow the flaps over time and see if these data correlate to the long-time survival of compromised flap areas. 
A limitation of this study was that the animals were not kept alive for a prolonged period after surgery, and we were therefore unable to monitor the compromised areas over time. In upcoming studies we will prospectively use LSCI to monitor flaps pre- and post-operatively to enable correlation analysis of the measurements with the outcome of the operation, and possibly identify threshold values for tissues at risk, that may be discarded at the time of surgery. Another limitation of the study is that we did not measure perfusion with either LSCI or LDF prior to surgery. These measurements would however probably not reflect the postoperative circulation of the flap, since the area would get part of its circulation from collateral vessels. It would also have been interesting to measure perfusion with LDF simultaneously in different regions of the flap, but we were unable to do this in the current study.

Although our results show clear benefits in favour of LSCI, in terms of variability and reliability, the technique has some limitations when compared to LDF. The LSCI apparatus is relatively bulky and, currently, more expensive than LDF. Because LSCI is a camera-based technique, it can only measure on tissues that are superficially exposed, such as radial flaps, ALT flaps, or to monitor replants. Also, even though data is acquired within seconds, the LSCI is highly sensitive to motion artefacts during this time, and misleading data can be obtained if the observer is inexperienced with the technique. LDF on the other hand collects data continuously and trends can easily be followed even by an inexperienced observer. Also, LDF allows for use on flaps were the flap surface is facing body cavities such as after intraoral reconstruction. For these reasons, LSCI is unlikely to replace LDF as the workhorse for postoperative free flap monitoring, at least in the near future, until some of the major limitations are resolved. The technique could instead be promising for assessment of the viability of different parts of skin flaps during and after surgery, to identify parts that may need to be discarded due to poor perfusion. 


\section{Acknowledgements:}

We thank Dan Linghammar at the Centre for Teaching and Research in Disaster Medicine and Traumatology, Linköping University Hospital for invaluable assistance with animal care and peroperative assistance.

\section{Conflicts of Interest}

None to declare

\section{Funding}

Funding was provided by County of Östergötland.

\section{Ethical Approval}

Ethical approval was given by the Regional Ethical Committee (Jordbruksverket) registration number 61-13. 


\section{References}

1. Smit, J.M., et al., Early reintervention of compromised free flaps improves success rate. Microsurgery, 2007. 27(7): p. 612-6.

2. Pattani, K.M., et al., What makes a good flap go bad? A critical analysis of the literature of intraoperative factors related to free flap failure. Laryngoscope, 2010. 120(4): p. 717-23.

3. Damen, T.H., et al., Improving outcomes in microsurgical breast reconstruction: lessons learnt from 406 consecutive DIEP/TRAM flaps performed by a single surgeon. J Plast Reconstr Aesthet Surg, 2013. 66(8): p. 1032-8.

4. Masoomi, H., et al., Predictive risk factors of free flap thrombosis in breast reconstruction surgery. Microsurgery, 2014. 34(8): p. 589-94.

5. Smit, J.M., et al., Advancements in free flap monitoring in the last decade: a critical review. Plast Reconstr Surg, 2010. 125(1): p. 177-85.

6. Binzoni, T., et al., Blood Perfusion Values of Laser Speckle Contrast Imaging and Laser Doppler Flowmetry: Is a Direct Comparision Possible? IEEE Transactions of Biomedical Engineering, 2013. 60(5): p. 1259-1265.

7. Clinton, M.S., et al., Establishment of normal ranges of laser Doppler blood flow in autologous tissue transplants. Plastic Reconstructive Surgery, 1991 Feb 87(2): p. 299-309.

8. Heller, L., L.S. Levin, and B. Klitzman, Laser Doppler flowmeter monitoring of free-tissue transfers: blood flow in normal and complicated cases. Plast. Reconstr. Surg., 2001. 107(7): p. 1739-45.

9. Zdolsek, J.M., et al., Laser Doppler perfusion imaging of the radial forearm flap: a clinical study. Scand J Plast Reconstr Surg Hand Surg, 2006. 40(2): p. 101-5.

10. Fredriksson, I., M. Larsson, and T. Stromberg, Measurement depth and volume in laser Doppler flowmetry. Microvasc Res, 2009. 78(1): p. 4-13.

11. Wardell, K., et al., Spatial heterogeneity in normal skin perfusion recorded with laser Doppler imaging and flowmetry. Microvasc Res, 1994. 48(1): p. 26-38.

12. Lindahl, F., E. Tesselaar, and F. Sjoberg, Assessing paediatric scald injuries using Laser Speckle Contrast Imaging. Burns, 2013. 39(4): p. 662-6.

13. Briers, D., et al., Laser speckle contrast imaging: theoretical and practical limitations. Journal of Biomedical Optics, 2013. 18(6): p. 0660018-1-9.

14. Roustit, M., et al., Excellent reproducibility of laser speckle contrast imaging to assess skin microvascular reactivity. Microvasc Res, 2010. 80(3): p. 505-11.

15. Tew, G.A., et al., Comparison of laser speckle contrast imaging with laser Doppler for assessing microvascular function. Microvasc Res, 2011. 82(3): p. 326-32.

16. Kroll, S.S., et al., Timing of pedicle thrombosis and flap loss after free tissue transfer. Plast Reconstr Surg, 1996. 98(7): p. 1230-33.

17. Russell, J.A., et al., Cutaneous tissue flap viability following partial venous obstruction. Plast Reconstr Surg, 2006. 117(7): p. 2259-66; discussion 2267-8.

18. Gimbel, M.L., et al., Monitoring partial and full venous outflow compromise in a rabbit skin flap model. Plast Reconstr Surg, 2009. 124(3): p. 796-803.

19. Meyer, W., R. Schwarz, and K. Neurand, The skin of domestic mammals as a model for the human skin, with special reference to the domestic pig. Curr Probl Dermatol, 1978. 7: p. 39-52.

20. Farnebo, S., et al., Hyperaemic changes in forearm skin perfusion and RBC concentration after increasing occlusion times. Microvasc Res, 2010. 80(3): p. 412-6. 


\section{Figure legends}

\section{Figure 1}

Above: Schematic overview of the surgical procedure of the cranial gluteal artery perforant (CGAP) flap and of the experimental protocol.

Below: Photographic montage showing a representative flap after it was raised. (A) The flap was designed large enough to contain three discrete zones (corresponding to regions examined with ROI 1-3 with LSCI), where zone 1 and 2 consistently were found to be well vascularized, and zone 3 was consistently poorly vascularized. (B) The flap was raised and (C) cuff and flow probes were placed around the supplying artery and the draining vein.(D) LDF probes and a temperature sensor were attached to the skin of the flap using double-adhesive tape.

Figure 2. Mean (SEM) \% change from baseline in perfusion in the well-vascularized part of the flap (ROI 1), for LSCI (circles) and LD (squares) for the six different phases of the protocol; (A) baseline, (B) 50\% venous occlusion, (C) 100\% venous occlusion, (D) recovery, (E) 100\% arterial occlusion and (F) recovery. Blood flow ( $\mathrm{ml} / \mathrm{min})$ through the supplying artery is presented with a dotted line and blood flow through the draining vein is presented with a thin grey line. 
Figure 3. LSCI images from one representative flap showing typical perfusion images during the six different phases of the protocol (left); (A) baseline, (B) 50\% venous occlusion, (C) 100\% venous occlusion, (D) recovery, (E) 100\% arterial occlusion and (F) recovery. Three different regions of interest (ROI 1-3) are indicated in the images, with decreasing perfusion depending on the distance from the pedicle, where ROI 1 and ROI 2 is in the well vascularized zone of the flap and ROI 3 is in the less vascularized zone (see Figure 1). (G) shows time course of the perfusion changes during these phases in the corresponding three regions of interest (ROI 1-3).

Figure 4. Perfusion in absolute AU (mean, SEM) as measured by LSCI in the well vascularized zone ROI 1, and the poorly vascularized zone ROI 3 for the six different phases of the protocol; (A) baseline, (B) 50\% venous occlusion, (C) 100\% venous occlusion, (D) recovery, (E) 100\% arterial occlusion and $(\mathrm{F})$ recovery. $* *(\mathrm{p}<0.005)$ and $*(<0.05)$ indicate that there is a significant difference in perfusion compared with baseline, ns $=$ no significant difference in perfusion compared with baseline.

Table 1. Mean (SD) blood pressure (systolic/diastolic), heart frequency and flap temperature over respective phase of the protocol.

Table 2. Changes in perfusion presented as means (SD) and \%CV\%. ** $(\mathrm{p}<0.005)$ and * $(<0.05)$ indicate that there is a significant difference in perfusion when compared to baseline, ns= non-significant difference in perfusion . 


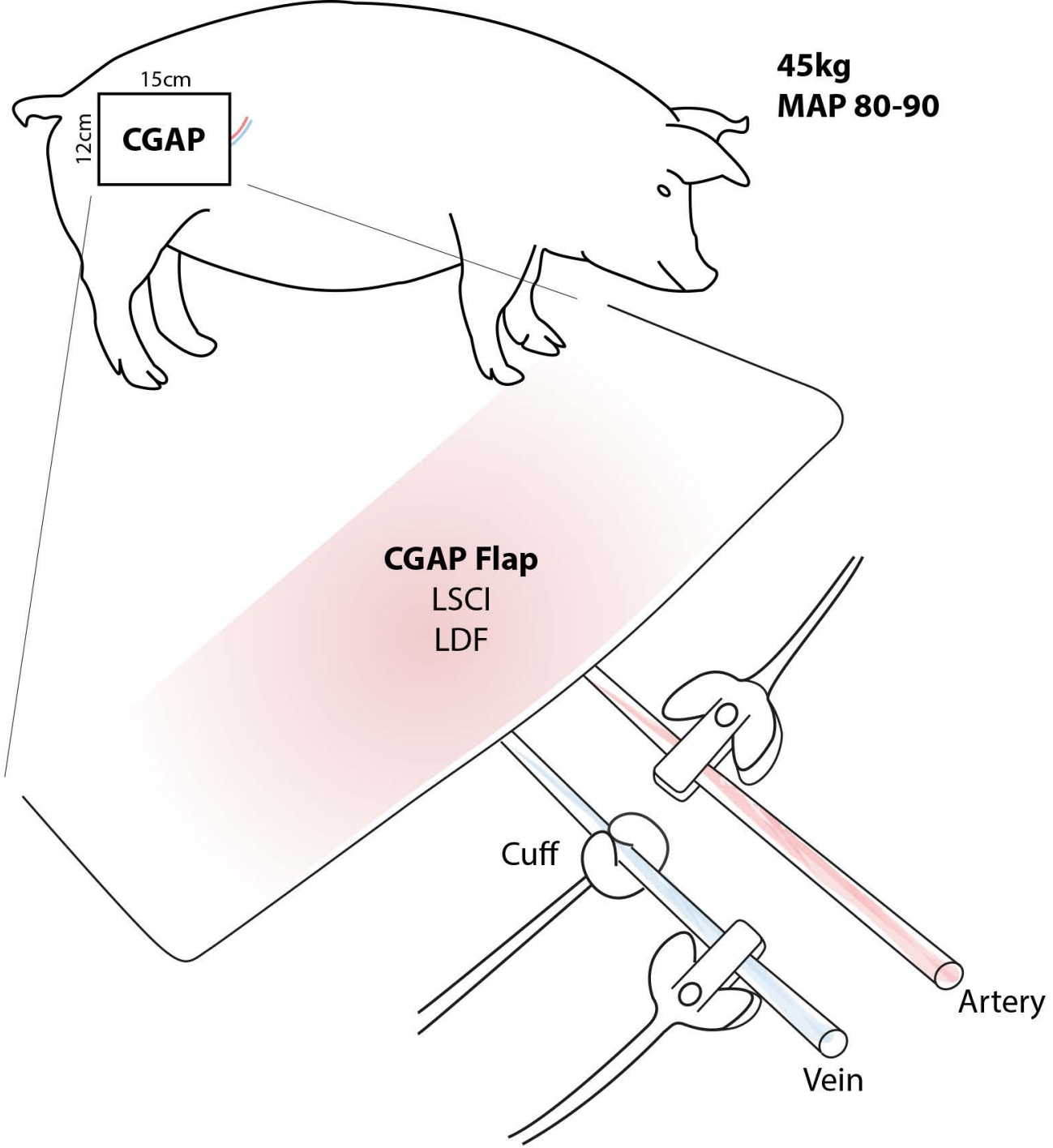

\section{Protocol:}

\begin{tabular}{|c|c|c|c|c|c|}
\hline $30 \mathrm{~min}$ & & $30 \mathrm{~min}$ & $40 \mathrm{~min}$ & $30 \mathrm{~min}$ & $30 \mathrm{~min}$ \\
\hline baseline & $\begin{array}{c}\mathbf{5 0 \%} \text { venous } \\
\text { stasis }\end{array}$ & $\begin{array}{c}100 \% \text { venous } \\
\text { stasis }\end{array}$ & recovery & $\begin{array}{c}\text { arterial } \\
\text { stasis }\end{array}$ & recovery \\
\hline
\end{tabular}

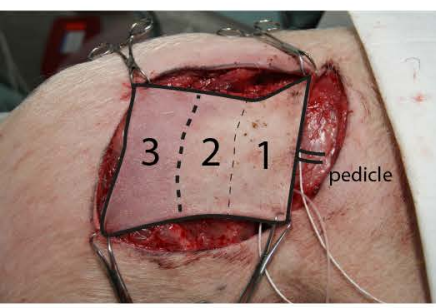

Regions of interest

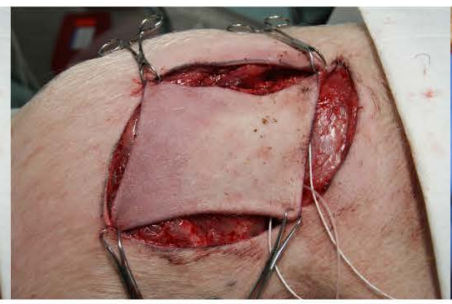

Flap raised

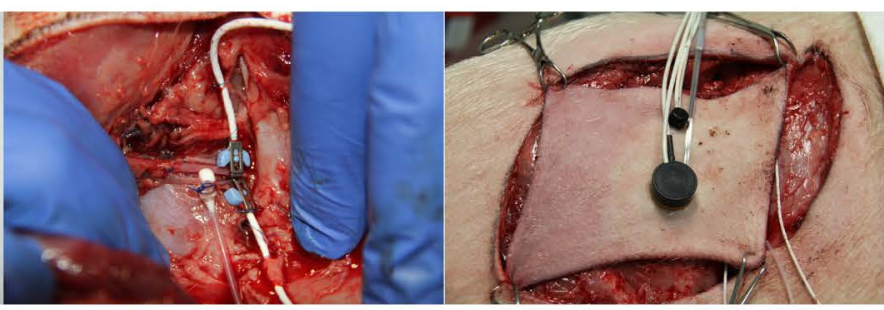

Pedicle with cuff and flowmeters
LDF probes and thermometer 


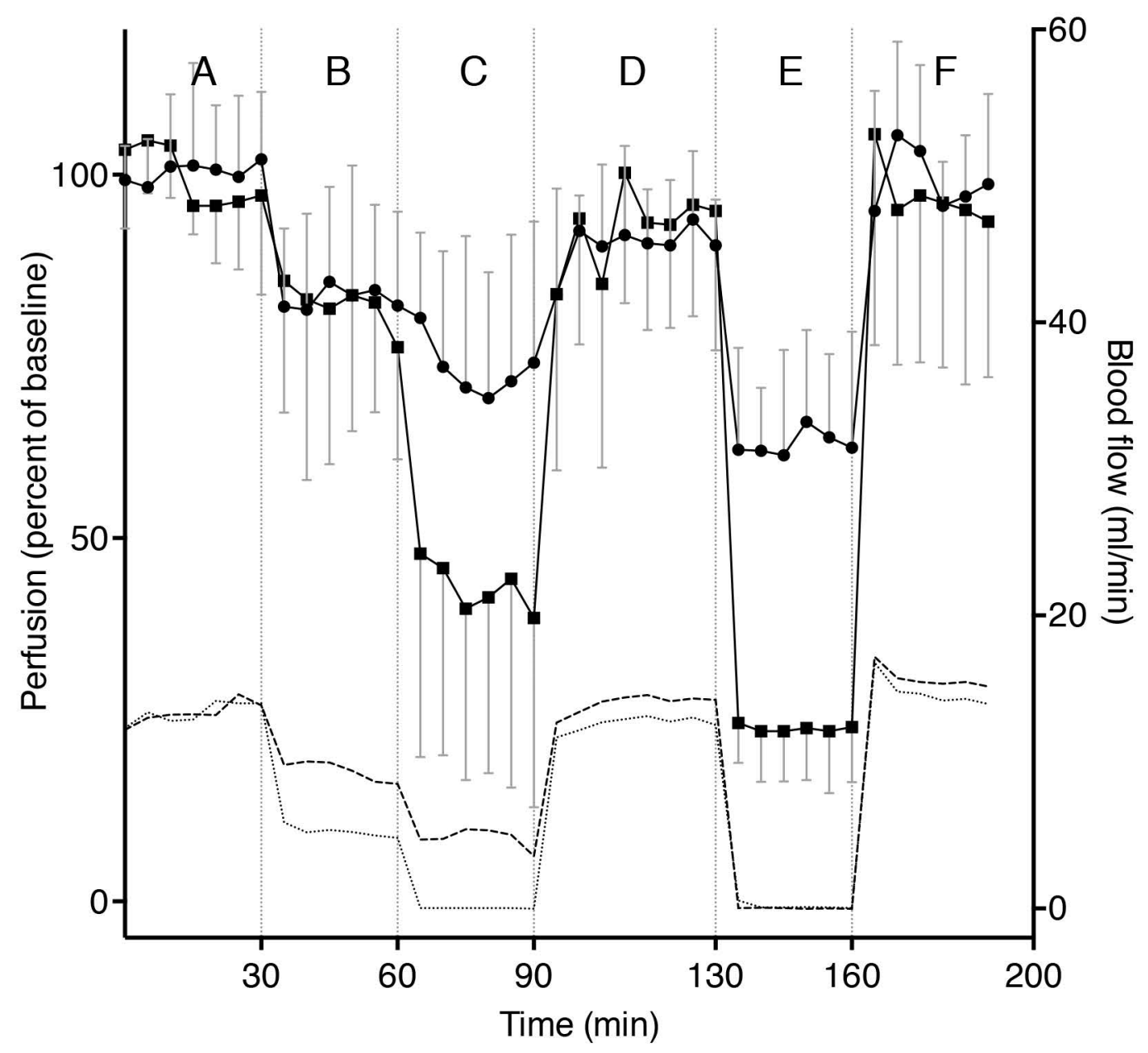

- $\mathrm{LSCl}$

$\rightarrow$ LD

---- Arterial

..... Venous 


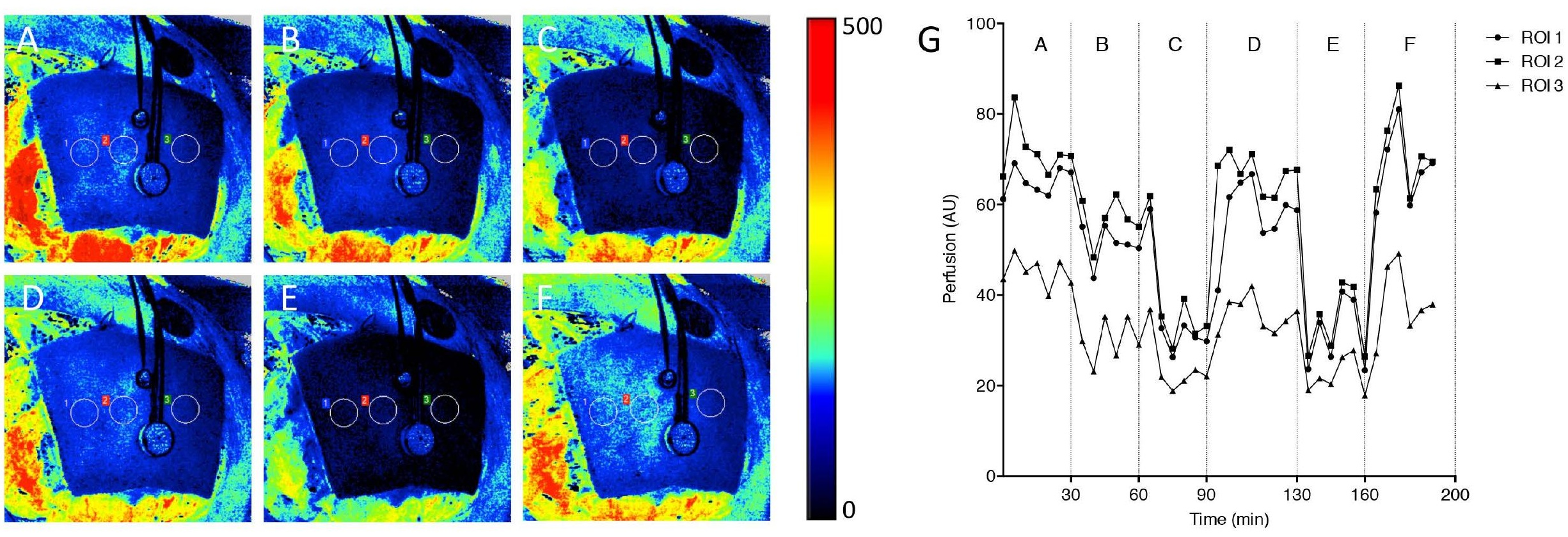




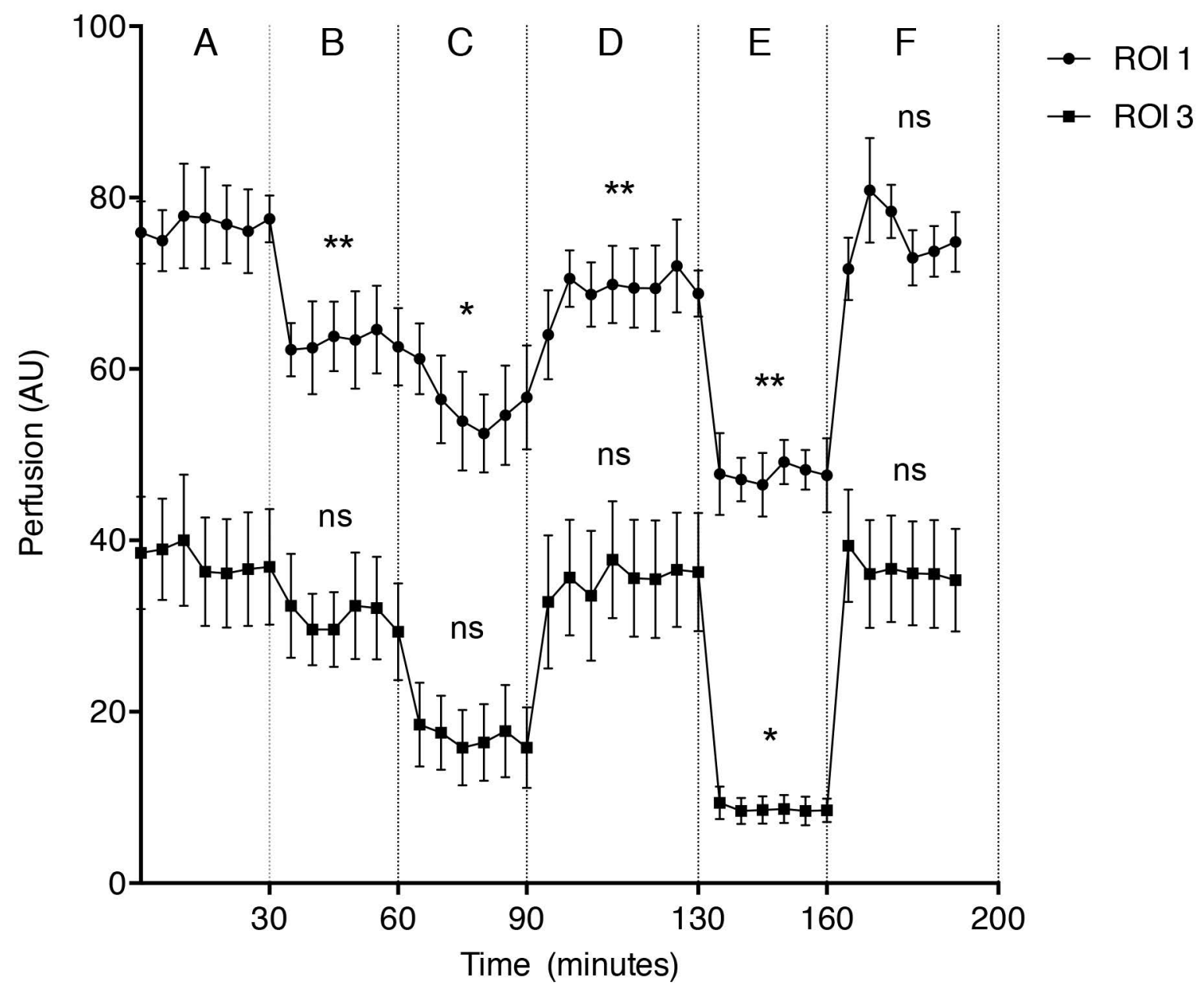


Table 1.

\section{Blood pressure}

mmHg; mean (SD)

Baseline

$50 \%$ venous occlusion

$100 \%$ venous occlusion

Recovery

$100 \%$ arterial occlusion

\section{Heart frequency}

Beats min-1; mean (SD)

$95(6)$

$95(6)$

$95(8)$

$95(5)$

$91(15)$

\section{Flap temperature}

degrees Celcius; mean (SD)

$39(0)$

$39(0)$

$40(1)$

$40(1)$

$40(0)$ 
Table 2.

\begin{tabular}{|c|c|c|c|c|}
\hline & \multicolumn{2}{|c|}{ LSCI } & \multicolumn{2}{|l|}{ LD } \\
\hline & mean (SD) & $\% \mathrm{CV}$ & mean (SD) & $\% \mathrm{CV}$ \\
\hline Baseline & $76.8(9.9)$ & 13 & $36.6(17.3)$ & 47 \\
\hline $50 \%$ venous occlusion & $63.5(12.9) * *$ & 20 & $31.3(15.7) \mathrm{ns}$ & 50 \\
\hline $100 \%$ venous occlusion & $54.6(14.2) * * *$ & 26 & $16.7(12.8) * * *$ & 77 \\
\hline Recovery & 70.1 (11.5) ns & 16 & 36.1 (17.9) ns & 50 \\
\hline $100 \%$ arterial occlusion & $48.3(7.7) * * *$ & 16 & $8.5(4.0) * * *$ & 45 \\
\hline
\end{tabular}

